\title{
Isometric deformations of isotropic surfaces
}

\author{
M. Dajczer and Th. Vlachos
}

\begin{abstract}
It was shown by Ramanathan [16] that any compact oriented minimal surface in the three-dimensional round sphere admits at most a finite set of pairwise noncongruent minimal isometric immersions. In this paper, we extend this result to isotropic surfaces in spheres of arbitrary dimension. The case of non-compact isotropic surfaces in space forms is also addressed.
\end{abstract}

It is standard fact that a simply-connected minimal surface $g: L^{2} \rightarrow \mathbb{Q}^{3}$ lying in a complete simply-connected three-dimensional space form possess an associated oneparameter family of minimal isometric deformations $g_{\theta}: L^{2} \rightarrow \mathbb{Q}^{3}, \theta \in \mathbb{S}^{1}=[0, \pi)$. On the other hand, if $L^{2}$ is non-simply-connected then the set of all minimal isometric deformations turns out to be either finite or still the full circle $\mathbb{S}^{1}$.

Of particular interest is when the ambient space is a sphere since only in that case the minimal surface $g: L^{2} \rightarrow \mathbb{S}^{3}$ is allowed to be compact. It was proved by Ramanathan [16] that a compact surface only allows finitely many minimal isometric deformations. Moreover, the surface must be equivariant, that is, any intrinsic one-parameter family of isometries becomes extrinsic, as is the case of the Clifford torus.

Our goal in this paper is to extend Ramanathan's results to any codimension. But, as already shown by him this can only be achieved under some strong additional condition on the surface. From the known results listed in $\S 2$, we can see why being isotropic comes in naturally for that purpose. In order to explain what for a surface being isotropic means, we need the concepts of higher fundamental forms and curvature ellipses that we briefly recall next and refer to $\S 1$ for details.

The normal bundle of a substantial minimal surface $g: L^{2} \rightarrow \mathbb{Q}^{N}$ splits orthogonally as the sum of plane bundles, except the last one which is a line bundle if the codimension is odd. These subbundles are spanned by the images of the higher fundamental forms. A minimal surface is called isotropic if the curvature ellipses of any order but the highest one in odd codimension, that is, the images of the unit tangent circle under the higher fundamental forms, are circles at any point of $L^{2}$. We observe that isotropic surfaces have been studied under various names in quite different circumstances, namely, as superminimal, superconformal and pseudoholomorphic surfaces.

It is well known that any simply-connected minimal surface $g: L^{2} \rightarrow \mathbb{Q}^{N}$ in any codimension also allows a one-parameter associated family $g_{\theta}: L^{2} \rightarrow \mathbb{Q}^{N}, \theta \in \mathbb{S}^{1}$, of 
isometric minimal immersions. The family is obtained integrating the system of Gauss, Codazzi and Ricci equations after rotating the second fundamental form a constant angle while keeping fixed the normal bundle and connection. If the surface is isotropic and lies in substantial even codimension, then the associated family is trivial in the sense that the surfaces are all pairwise congruent. On the other hand, in substantial odd codimension the associated family in never trivial. Moreover, all surfaces in the associated family are isotropic and there are no other isotropic ones.

Isotropic surfaces have been frequently considered in the literature. The most obvious examples are holomorphic curves in $\mathbb{C}^{N}$ that can also be seen as isotropic surfaces in $\mathbb{R}^{2 N}$. As for simply-connected isotropic surfaces in Euclidean space with odd codimension there is a Weierstrass type representation given in [7] that generates all of them.

When the ambient space is a round sphere, it results from the works of Calabi [6] and Barbosa [1] that any topological 2-sphere minimally immersed in $\mathbb{S}^{N}$ is isotropic and lies in even substantial codimension. Miyaoka [15] described the simply-connected isotropic surfaces in spheres with odd codimension in terms of the solutions of the affine Toda equation and, as an application, managed to give all flat minimal tori in an explicit parametric form.

It was shown by Vlachos [18] that the so called Lawson's surfaces in spheres are isotropic but only for certain values of the parameters. Examples of isotropic surfaces in $\mathbb{S}^{5}$ that include nonflat compact tori, came out from Bryant's [5] theory of pseudoholomorphic surfaces in the nearly Kaehler $\mathbb{S}^{6}$; see also [2, [4],12] and [13]. Finally, observe that the polar surface (to be defined in $§ 2$ ) associated to an isotropic substantial surface in an odd dimensional sphere is also isotropic.

Our first result is of local nature and follows from a quite simple argument.

Theorem 1. Let $g: L^{2} \rightarrow \mathbb{Q}^{2 n+1}$ be an oriented isotropic substantial surface. Then, the set of all isotropic immersions of $L^{2}$ into $\mathbb{Q}^{2 n+1}$ is either finite or a circle.

For the case of compact surfaces we have the main result of the paper.

Theorem 2. Let $g: L^{2} \rightarrow \mathbb{S}^{2 n+1}$ be an oriented isotropic substantial surface. If $L^{2}$ is compact, then there exist at most finitely many isotropic immersions of $L^{2}$ into $\mathbb{S}^{2 n+1}$.

The following is an immediate consequence of the last result.

Corollary 3. Let $g: L^{2} \rightarrow \mathbb{S}^{2 n+1}$ be an oriented compact isotropic substantial surface. If $L^{2}$ admits a continuous one-parameter family of isometries $\varphi_{t}$ with $\varphi_{0}=i d$, then there exists a continuous one-parameter family of isometries $\tau_{t}$ of $\mathbb{S}^{2 n+1}$ such that $g \circ \varphi_{t}=\tau_{t} \circ g$ for any value of the parameter. 


\section{Preliminaries}

In this section, we collect several facts and definitions about minimal surfaces in space forms. For some of the details we refer to [9] and [11].

Let $g: L^{2} \rightarrow \mathbb{Q}^{N}$ denote an isometric immersion of a two-dimensional Riemannian manifold. The $k^{\text {th }}$-normal space of $g$ at $x \in L^{2}$ for $k \geq 1$ is defined as

$$
N_{k}^{g}(x)=\operatorname{span}\left\{\alpha_{g}^{k+1}\left(X_{1}, \ldots, X_{k+1}\right): X_{1}, \ldots, X_{k+1} \in T_{x} L\right\}
$$

where

$$
\alpha_{g}^{s}: T L \times \cdots \times T L \rightarrow N_{g} L, \quad s \geq 3
$$

denotes the symmetric tensor called the $s^{\text {th }}$-fundamental form given inductively by

$$
\alpha_{g}^{s}\left(X_{1}, \ldots, X_{s}\right)=\left(\nabla_{X_{s}}^{\perp} \ldots \nabla_{X_{3}}^{\perp} \alpha_{g}\left(X_{2}, X_{1}\right)\right)^{\perp}
$$

and $\alpha_{g}: T L \times T L \rightarrow N_{g} L$ stands for the standard second fundamental form of $g$ with values in the normal bundle. Here $\nabla^{\perp}$ denotes the induced connection in the normal bundle $N_{g} L$ of $g$ and ()$^{\perp}$ means taking the projection onto the normal complement of $N_{1}^{g} \oplus \ldots \oplus N_{s-2}^{g}$ in $N_{g} L$.

Let $g: L^{2} \rightarrow \mathbb{Q}^{N}$ be a minimal isometric immersion. If $L^{2}$ is simply-connected, there exists a one-parameter associated family of minimal isometric immersions $g_{\theta}: L^{2} \rightarrow \mathbb{Q}^{N}$, $\theta \in \mathbb{S}^{1}=[0, \pi)$ with real-analytic dependence on the parameter. To see this, for each $\theta \in \mathbb{S}^{1}$ consider the orthogonal parallel tensor field

$$
J_{\theta}=\cos \theta I+\sin \theta J
$$

where $I$ is the identity map. Then, the symmetric section $\alpha_{g}\left(J_{\theta} \cdot, \cdot\right)$ of the bundle $\operatorname{Hom}\left(T L \times T L, N_{g} L\right)$ satisfies the Gauss, Codazzi and Ricci equations with respect to the normal bundle and normal connection of $g$; see [10] for details. Therefore, there exists an isometric minimal immersion $g_{\theta}: L^{2} \rightarrow \mathbb{Q}^{N}$ whose second fundamental form is

$$
\alpha_{g_{\theta}}(X, Y)=\phi_{\theta} \alpha_{g}\left(J_{\theta} X, Y\right)
$$

where $\phi_{\theta}: N_{g} L \rightarrow N_{g_{\theta}} L$ is the parallel vector bundle isometry that identifies the normal subbundles $N_{j}^{g}$ with $N_{j}^{g_{\theta}}, j \geq 1$.

A surface $g: L^{2} \rightarrow \mathbb{Q}^{N}$ is called regular if for each $k$ the subspaces $N_{k}^{g}$ have constant dimension and thus form normal subbundles. Notice that regularity is always verified along connected components of an open dense subset of $L^{2}$.

Assume that an immersion $g: L^{2} \rightarrow \mathbb{Q}^{N}$ is minimal and substantial. The latter condition means that the codimension cannot be reduced. In this case, the normal bundle of $g$ splits along the open dense subset of $L^{2}$ of regular points as

$$
N_{g} L=N_{1}^{g} \oplus N_{2}^{g} \oplus \cdots \oplus N_{m}^{g}, \quad m=[(N-1) / 2],
$$


since all higher normal bundles have rank two except possible the last one that has rank one if $N$ is odd; see [8] or [9]. Moreover, if $L^{2}$ is oriented, then an orientation is induced on each plane vector bundle $N_{s}^{g}$ given by the ordered base

$$
\xi_{1}^{s}=\alpha_{g}^{s+1}(X, \ldots, X), \quad \xi_{2}^{s}=\alpha_{g}^{s+1}(J X, \ldots, X)
$$

where $0 \neq X \in T L$ and $J$ is the complex structure of $L^{2}$ determined by the metric and the orientation.

If $g: L^{2} \rightarrow \mathbb{Q}^{N}$ is regular, then at $x \in L^{2}$ and for each $N_{k}^{g}, 1 \leq k \leq m$, the $k^{\text {th }}$-order curvature ellipse $\mathcal{E}_{k}^{g}(x) \subset N_{k}^{g}(x)$ is defined by

$$
\mathcal{E}_{k}^{g}(x)=\left\{\alpha_{g}^{k+1}\left(Z^{\varphi}, \ldots, Z^{\varphi}\right): Z^{\varphi}=\cos \varphi Z+\sin \varphi J Z \text { and } \varphi \in \mathbb{S}^{1}\right\}
$$

where $Z \in T_{x} L$ is any vector of unit length.

A regular surface $g: L^{2} \rightarrow \mathbb{Q}^{N}$ is called isotropic if it is minimal and at any $x \in L^{2}$ the ellipses of curvature $\mathcal{E}_{k}^{g}(x)$ contained in all two-dimensional $N_{k}^{g}$ 's are circles. We point out that there are alternative ways to define isotropy for surfaces, for instance, in terms of the vanishing of higher order Hopf differentials [17] or as the mutual orthogonality of the complex line bundles determined by the harmonic sequences associated to the surface [3].

\section{Isotropic surfaces}

In this section, we list several properties of isotropic surfaces in space forms already part of the literature. They may just justify the assumptions of our results or go further as to be ingredients of the proofs.

The first result follows from the arguments of Chern in [8] and has been proved in Proposition 4 of [18].

Fact 4. If $g: L^{2} \rightarrow \mathbb{Q}^{N}$ is an isotropic surface, then the set $L_{0}$ where $g$ fails to be regular is formed of isolated points and all $N_{k}^{g}$ 's extend smoothly to $L_{0}$.

From either Corollary 6.1 in [14] or Corollary 5.2 in [17] or Theorem 2 in [11] we have the following result.

Fact 5. Any pair of isometric isotropic surfaces in $\mathbb{Q}^{N}$ that are substantial in even codimension are congruent.

The situation for odd codimension is different since Theorem 2 in [11] gives the following result for associated families. 
Fact 6. Surfaces belonging to the associated family of a simply-connected isotropic surface in $\mathbb{Q}^{N}$ that is substantial in odd codimension are pairwise noncongruent.

For a simply-connected isotropic surface in odd substantial codimension first notice that it follows from the definition that taking the associated family preserves isotropy. The following result is due to Johnson [14]; see Vlachos [17] for another proof.

Fact 7. If $g: L^{2} \rightarrow \mathbb{Q}^{2 n+1}$ is an oriented simply-connected isotropic substantial surface, then any other isotropic substantial isometric immersion $f: L^{2} \rightarrow \mathbb{Q}^{2 n+1}$ belongs to the associated family $g_{\theta}$ of $g$.

Given a non-simply-connected isotropic oriented substantial surface $g: L^{2} \rightarrow \mathbb{Q}^{2 n+1}$ let $f: L^{2} \rightarrow \mathbb{Q}^{2 n+1}$ be another isotropic isometric substantial surface. Let $\pi: \tilde{L}^{2} \rightarrow L^{2}$ denote the universal covering map with $\tilde{L}^{2}$ equipped with the metric and orientation that makes $\pi$ an orientation preserving local isometry. In the sequel, we denote corresponding objects on $\tilde{L}^{2}$ with a tilde. Since the surfaces $\tilde{g}=g \circ \pi$ and $\tilde{f}=f \circ \pi$ are isotropic, it follows from Fact 7 that $\tilde{f}$ is congruent to some $\tilde{g}_{\theta}$ in the associated family of $\tilde{g}$. We thus have the following consequence.

Fact 8. Let $g: L^{2} \rightarrow \mathbb{Q}^{2 n+1}$ be an oriented non-simply-connected isotropic substantial surface. Then, the set

$$
\mathcal{M}(g)=\left\{\theta \in \mathbb{S}^{1}: \text { there is } f: L^{2} \rightarrow \mathbb{Q}^{2 n+1} \text { such that } f=\tilde{g}_{\theta} \circ \pi\right\}
$$

parametrizes the space of isometric isotropic substantial immersions of $L^{2}$ into $\mathbb{Q}^{2 n+1}$.

Let $g: L^{2} \rightarrow \mathbb{S}^{2 n+1}$ be an isotropic substantial surface and consider a smooth unit vector field $e \in N_{g} L$ such that $\operatorname{span}\{e\}=N_{n}^{g}$. The surface $g^{*}=e: L^{2} \backslash L_{0} \rightarrow \mathbb{S}^{2 n+1}$ is usually called the polar surface to $g$. The first statement in the next result follows from Proposition 8 in [9] whereas the second statement follows from Proposition 2 and Theorem 2 in [19].

Fact 9. The polar surface $g^{*}: L^{2} \backslash L_{0} \rightarrow \mathbb{S}^{2 n+1}$ to a given oriented isotropic substantial surface $g: L^{2} \rightarrow \mathbb{S}^{2 n+1}$ is also isotropic. Moreover, the metric induced by $g^{*}$ is conformal to the metric of $L^{2}$ by a conformal factor that depends only on the metric of $L^{2}$.

\section{The proofs}

In this section, we provide the proofs of our results stated in the introduction.

We first observe the following elementary fact. 
Proposition 10. Let $g: L^{2} \rightarrow \mathbb{Q}^{N}$ be a non-simply-connected isotropic surface. For any $\sigma \in \mathcal{D}$ in the group of deck transformations of $\pi: \tilde{L}^{2} \rightarrow L^{2}$ the surfaces $\tilde{g}_{\theta}: \tilde{L}^{2} \rightarrow \mathbb{Q}^{N}$ and $\tilde{g}_{\theta} \circ \sigma: \tilde{L}^{2} \rightarrow \mathbb{Q}^{N}$ are congruent for any $\theta \in \mathbb{S}^{1}$.

Proof: It is sufficient to show the existence of a parallel vector bundle isometry between the normal bundles of $\tilde{g}_{\theta}$ and $\tilde{g}_{\theta} \circ \sigma$ that preserves the second fundamental forms. Let $\phi_{\theta}$ be the vector isometry between the normal bundles of $\tilde{g}$ and $\tilde{g}_{\theta}$. Define a vector bundle isometry $\psi_{\theta}: N_{\tilde{g}_{\theta}} \tilde{L} \rightarrow N_{\tilde{g}_{\theta} \circ \sigma} \tilde{L}$ by

$$
\psi_{\theta} \xi=\phi_{\theta}\left(\eta \circ \sigma^{-1}\right) \circ \sigma
$$

where $\xi=\phi_{\theta} \eta$ for $\eta \in N_{\tilde{g}} \tilde{L}$. The second fundamental forms of $\tilde{g}_{\theta} \circ \sigma$ and $\tilde{g}_{\theta}$ relate by

$$
\alpha_{\tilde{g}_{\theta} \circ \sigma}(\tilde{X}, \tilde{Y})=\phi_{\theta} \alpha_{\tilde{g}}\left(\tilde{J}_{\theta} \sigma_{*} \tilde{X}, \sigma_{*} \tilde{Y}\right)
$$

for any $\tilde{X}, \tilde{Y} \in T \tilde{L}$, where

$$
\tilde{J}_{\theta}=\cos \theta \tilde{I}+\sin \theta \tilde{J} .
$$

Since $\sigma$ is a deck transformation, then

$$
\alpha_{\tilde{g}_{\theta} \circ \sigma}=\psi_{\theta} \circ \alpha_{\tilde{g}_{\theta}} .
$$

Let $\xi=\phi_{\theta} \eta$ where $\eta \in N_{\tilde{g}} \tilde{L}$. We have that

$$
\begin{aligned}
\left(\nabla_{\tilde{X}}^{\perp} \psi_{\theta}\right) \xi & =\nabla_{\tilde{X}}^{\perp}\left(\phi_{\theta}\left(\eta \circ \sigma^{-1}\right) \circ \sigma\right)-\phi_{\theta}\left(\nabla_{\tilde{X}}^{\perp}\left(\eta \circ \sigma^{-1}\right)\right) \circ \sigma \\
& =\left(\nabla_{\sigma_{*} \tilde{X}}^{\perp} \phi_{\theta}\left(\eta \circ \sigma^{-1}\right)\right) \circ \sigma-\phi_{\theta}\left(\nabla_{\tilde{X}}^{\perp}\left(\eta \circ \sigma^{-1}\right)\right) \circ \sigma \\
& =\phi_{\theta}\left(\nabla_{\sigma_{*} \tilde{X}}^{\perp}\left(\eta \circ \sigma^{-1}\right)-\nabla_{\tilde{X}}^{\perp}\left(\eta \circ \sigma^{-1}\right)\right) \circ \sigma
\end{aligned}
$$

where $\tilde{X} \in T \tilde{L}$ and $\nabla^{\perp}$ stands for the normal connection of $\tilde{g}_{\theta}$ as well as for $\tilde{g}_{\theta} \circ \sigma$. Now let $\delta \in N_{g} L$ be such that $\eta \circ \sigma^{-1}=\delta \circ \pi$. Observe that

$$
\nabla_{\sigma_{*} \tilde{X}}^{\perp} \eta \circ \sigma^{-1}-\nabla_{\tilde{X}}^{\perp} \eta \circ \sigma^{-1}=\nabla_{\pi_{*} \sigma_{*} \tilde{X}}^{\perp} \delta-\nabla_{\pi_{*} \tilde{X}}^{\perp} \delta=0,
$$

and thus $\psi_{\theta}$ is parallel.

Proof of Theorem 1]: Proposition 10 yields a map $\Phi_{\theta}: \mathcal{D} \rightarrow \operatorname{Isom}\left(\mathbb{Q}^{2 n+1}\right)$ for each given $\theta \in \mathbb{S}^{1}$ such that

$$
\tilde{g}_{\theta} \circ \sigma=\Phi_{\theta}(\sigma) \circ \tilde{g}_{\theta} .
$$

Thus $\theta \in \mathcal{M}(g)$ if and only if $\Phi_{\theta}(\mathcal{D})=\{I\}$. Suppose that $\mathcal{M}(g)$ is not finite and let $\left\{\theta_{m}\right\}_{m \in \mathbb{N}}$ be a sequence in $\mathcal{M}(g)$ that converges to some $\theta_{0} \in \mathbb{S}^{1}$. Hence $\Phi_{\theta_{0}}(\mathcal{D})=\{I\}$.

Take $\sigma \in \mathcal{D}$. By the Mean value Theorem applied to each entry $\left(\Phi_{\theta}(\sigma)\right)_{j k}$ of the corresponding matrix, we have

$$
\frac{d}{d \theta}\left(\Phi_{\theta}(\sigma)\right)_{j k}\left(\stackrel{\circ}{m}_{m}\right)=0
$$


for some $\stackrel{\circ}{m}_{m}$ which lies between $\theta_{0}$ and $\theta_{m}$. By continuity it follows that

$$
\frac{d}{d \theta}\left(\Phi_{\theta}(\sigma)\right)_{j k}\left(\theta_{0}\right)=0
$$

Consider the sequence $\left\{\AA_{m}\right\}_{m \in \mathbb{N}}$ that converges $\theta_{0}$ and observe that in view of (2) similar argument gives

$$
\frac{d^{2}}{d \theta^{2}}\left(\Phi_{\theta}(\sigma)\right)_{j k}\left(\theta_{0}\right)=0
$$

Repeating the argument yields

$$
\frac{d^{n}}{d \theta^{n}}\left(\Phi_{\theta}(\sigma)\right)_{j k}\left(\theta_{0}\right)=0
$$

for any integer $n \geq 1$. Since $\Phi_{\theta}(\sigma)$ is an analytic curve in $\operatorname{Isom}\left(\mathbb{Q}^{2 n+1}\right)$, we conclude that $\Phi_{\theta}(\sigma)=I$ for each $\sigma \in \mathcal{D}$ and Fact 8 now gives $\mathcal{M}(g)=\mathbb{S}^{1}$.

The proof of the following fact is immediate.

Lemma 11. Let $g: L^{2} \rightarrow \mathbb{Q}^{2 n+1}$ be an isotropic substantial surface. For any $\theta \in \mathcal{M}(g)$ there exists a parallel vector bundle isometry $\phi_{\theta}: N_{g} L \rightarrow N_{\tilde{g}_{\theta} \circ \pi} L$ such that the higher fundamental forms of $g$ and $\tilde{g}_{\theta} \circ \pi$ are related by

$$
\alpha_{\tilde{g}_{\theta} \circ \pi}^{s}\left(X_{1}, \ldots, X_{s}\right)=\phi_{\theta} \alpha_{g}^{s}\left(J_{\theta} X_{1}, \ldots, X_{s}\right)
$$

where $X_{1}, \ldots, X_{s} \in T L$.

Proof of Theorem 2: Choose a positively oriented orthonormal frame $\left\{e_{1}, e_{2}\right\}$ in $T L$ along an open subset of $L^{2} \backslash L_{0}$. Since $g$ is isotropic, we may choose a local orthonormal normal frame $\left\{e_{3}, \ldots, e_{2 n+1}\right\}$ such that $\left\{e_{2 s+1}, e_{2 s+2}\right\}$ is given by

$$
\alpha_{g}^{s+1}\left(e_{1}, \ldots, e_{1}, e_{1}\right)=\kappa_{s} e_{2 s+1} \text { and } \alpha_{g}^{s+1}\left(e_{1}, \ldots, e_{1}, e_{2}\right)=\kappa_{s} e_{2 s+2}, \quad 1 \leq s \leq n-1
$$

where $\kappa_{s}$ denotes the radii of the circular ellipse of curvature $\mathcal{E}_{s}^{g}$. Then $e_{2 n+1}$ spans the last normal bundle. According to Lemma 5 in [18] the normal connection forms

$$
\omega_{\alpha \beta}=\left\langle\nabla^{\perp} e_{\alpha}, e_{\beta}\right\rangle
$$

satisfy

$$
\begin{gathered}
\omega_{2 s, 2 s+1}=-* \omega_{2 s-1,2 s+1}, \quad \omega_{2 s, 2 s+2}=-* \omega_{2 s-1,2 s+2}, \\
\omega_{2 s-1,2 s+2}=* \omega_{2 s-1,2 s+1}, \quad \omega_{2 s, 2 s+2}=* \omega_{2 s, 2 s+1}, \quad 1 \leq s \leq n-1,
\end{gathered}
$$

and

$$
\omega_{2 n, 2 n+1}=-* \omega_{2 n-1,2 n+1}
$$

where $*$ denotes the Hodge operator, i.e., $* \omega=-\omega \circ J$. 
The metric and second fundamental of $g$ can be complex linearly extended to the complexified tangent bundle $T L \otimes \mathbb{C}$ and complexified normal bundle $N_{g} L \otimes \mathbb{C}$. Setting $E=e_{1}-i e_{2}$, then (3), (41) and (5) yield

$$
\begin{gathered}
\omega_{2 s-1,2 s+2}(E)=-i \omega_{2 s-1,2 s+1}(E), \quad \omega_{2 s, 2 s+1}(E)=i \omega_{2 s-1,2 s+1}(E), \\
\omega_{2 s, 2 s+2}(E)=\omega_{2 s-1,2 s+1}(E), \quad 1 \leq s \leq n-1
\end{gathered}
$$

and

$$
\omega_{2 n, 2 n+1}(E)=i \omega_{2 n-1,2 n+1}(E) .
$$

Take $\theta_{1}<\cdots<\theta_{m} \in \mathcal{M}(g)$. In the sequel, we regard the surfaces $g_{\theta_{j}}=\tilde{g}_{\theta_{j}} \circ \pi$ as lying in $\mathbb{R}^{2 n+2}$ by composing with the umbilical inclusion $\mathbb{S}^{2 n+1} \hookrightarrow \mathbb{R}^{2 n+2}$. We claim that if

$$
\sum_{j=1}^{m}\left\langle g_{\theta_{j}}, v_{j}\right\rangle=0
$$

for some $v_{j} \in \mathbb{R}^{2 n+2}$, then $v_{j}=0$ for all $1 \leq j \leq m$.

To prove the claim, we may assume that $v_{j} \neq 0$ for all $1 \leq j \leq m$. Differentiating (9) yields

$$
\sum_{j=1}^{m}\left\langle g_{\theta_{j_{*}}}, v_{j}\right\rangle=0 \text { and } \sum_{j=1}^{m}\left\langle\alpha_{g_{\theta_{j}}}, v_{j}\right\rangle=0 .
$$

Then, Lemma 11 and the second equation in (10) give

$$
\sum_{j=1}^{m}\left\langle\phi_{\theta_{j}} \alpha_{g}\left(J_{\theta} E, E\right), v_{j}\right\rangle=0
$$

Since $J_{\theta} E=e^{i \theta} E$, it follows easily that

$$
\sum_{j=1}^{m} e^{i \theta_{j}}\left\langle\phi_{\theta_{j}}\left(e_{3}-i e_{4}\right), v_{j}\right\rangle=0
$$

Differentiating (11) with respect to $X \in T M \otimes \mathbb{C}$ yields

$$
\sum_{j=1}^{m} e^{i \theta_{j}}\left\langle g_{\theta_{j_{*}}} A_{\phi_{\theta_{j}}\left(e_{3}-i e_{4}\right)} X, v_{j}\right\rangle-\sum_{j=1}^{m} e^{i \theta_{j}}\left\langle\nabla_{X}^{\perp} \phi_{\theta_{j}}\left(e_{3}-i e_{4}\right), v_{j}\right\rangle=0
$$

where $A_{\phi_{\theta_{j}} \eta}$ is the shape operator of $g_{\theta_{j}}$ in the direction $\phi_{\theta_{j}} \eta$. Lemma 11] and (11) give

$$
A_{\phi_{\theta_{j}}\left(e_{3}-i e_{4}\right)}=e^{-i \theta_{j}} A_{e_{3}-i e_{4}}
$$


where $A_{\eta}$ denotes the shape operator of $g$. This and the first equation in (10) yield

$$
\sum_{j=1}^{m} e^{i \theta_{j}}\left\langle\nabla_{X}^{\perp} \phi_{\theta_{j}}\left(e_{3}-i e_{4}\right), v_{j}\right\rangle=0
$$

In view of (11) this equation is equivalent to

$$
\left(\omega_{35}(X)-i \omega_{45}(X)\right) \sum_{j=1}^{m} e^{i \theta_{j}}\left\langle\phi_{\theta_{j}} e_{5}, v_{j}\right\rangle+\left(\omega_{36}(X)-i \omega_{46}(X)\right) \sum_{j=1}^{m} e^{i \theta_{j}}\left\langle\phi_{\theta_{j}} e_{6}, v_{j}\right\rangle=0 .
$$

On the other hand, we obtain from (6) and (7) that

$$
\omega_{45}(E)=i \omega_{35}(E), \omega_{36}(E)=-i \omega_{35}(E) \text { and } \omega_{46}(E)=\omega_{35}(E) .
$$

Using (13) it follows that (12) for $X=E$ becomes

$$
\omega_{35}(E) \sum_{j=1}^{m} e^{i \theta_{j}}\left\langle\phi_{\theta_{j}}\left(e_{5}-i e_{6}\right), v_{j}\right\rangle=0
$$

We have from (13) that $\omega_{35}(E)$ cannot vanish since $g$ is substantial. Hence,

$$
\sum_{j=1}^{m} e^{i \theta_{j}}\left\langle\phi_{\theta_{j}}\left(e_{5}-i e_{6}\right), v_{j}\right\rangle=0
$$

We argue that

$$
\sum_{j=1}^{m} e^{i \theta_{j}}\left\langle\phi_{\theta_{j}}\left(e_{2 s+1}-i e_{2 s+2}\right), v_{j}\right\rangle=0, \quad s \leq n-1
$$

and

$$
\sum_{j=1}^{m} e^{i \theta_{j}}\left\langle\phi_{\theta_{j}} e_{2 n+1}, v_{j}\right\rangle=0
$$


By induction, we assume (14) for $s=r$ and differentiate with respect to $E$. We obtain

$$
\begin{aligned}
\left(i \omega_{2 r-1,2 r+2}(E)\right. & \left.-\omega_{2 r-1,2 r+1}(E)\right) \sum_{j=1}^{m} e^{i \theta_{j}}\left\langle\phi_{\theta_{j}} e_{2 r-1}, v_{j}\right\rangle \\
& +\left(i \omega_{2 r, 2 r+2}(E)-\omega_{2 r, 2 r+1}(E)\right) \sum_{j=1}^{m} e^{i \theta_{j}}\left\langle\phi_{\theta_{j}} e_{2 r}, v_{j}\right\rangle \\
& +i \omega_{2 r+1,2 r+2}(E) \sum_{j=1}^{m} e^{i \theta_{j}}\left\langle\phi_{\theta_{j}}\left(e_{2 r+1}-i e_{2 r+2}\right), v_{j}\right\rangle \\
& +\left(\omega_{2 r+1,2 r+3}(E)-i \omega_{2 r+2,2 r+3}(E)\right) \sum_{j=1}^{m} e^{i \theta_{j}}\left\langle\phi_{\theta_{j}} e_{2 r+3}, v_{j}\right\rangle \\
& +\left(\omega_{2 r+1,2 r+4}(E)-i \omega_{2 r+2,2 r+4}(E)\right) \sum_{j=1}^{m} e^{i \theta_{j}}\left\langle\phi_{\theta_{j}} e_{2 r+4}, v_{j}\right\rangle \\
& =0
\end{aligned}
$$

if $r \leq n-2$. If $r=n-1$, we have

$$
\begin{aligned}
\left(i \omega_{2 n-3,2 n}(E)\right. & \left.-\omega_{2 n-3,2 n-1}(E)\right) \sum_{j=1}^{m} e^{i \theta_{j}}\left\langle\phi_{\theta_{j}} e_{2 n-3}, v_{j}\right\rangle \\
& +\left(i \omega_{2 n-2,2 n}(E)-\omega_{2 n-2,2 n-1}(E)\right) \sum_{j=1}^{m} e^{i \theta_{j}}\left\langle\phi_{\theta_{j}} e_{2 n-2}, v_{j}\right\rangle \\
& +i \omega_{2 n-1,2 n}(E) \sum_{j=1}^{m} e^{i \theta_{j}}\left\langle\phi_{\theta_{j}}\left(e_{2 n-1}-i e_{2 n}\right), v_{j}\right\rangle \\
& +\left(\omega_{2 n-1,2 n+1}(E)-i \omega_{2 n, 2 n+1}(E)\right) \sum_{j=1}^{m} e^{i \theta_{j}}\left\langle\phi_{\theta_{j}} e_{2 n+1}, v_{j}\right\rangle \\
& =0 .
\end{aligned}
$$

Using (6), (17) and (8), we obtain

$$
\omega_{2 r+1,2 r+3}(E) \sum_{j=1}^{m} e^{i \theta_{j}}\left\langle\phi_{\theta_{j}}\left(e_{2 r+3}-i e_{2 r+4}\right), v_{j}\right\rangle=0, \quad r \leq n-2,
$$

and

$$
\omega_{2 n-1,2 n+1}(E) \sum_{j=1}^{m} e^{i \theta_{j}}\left\langle\phi_{\theta_{j}} e_{2 n+1}, v_{j}\right\rangle=0 .
$$

Since $g$ is substantial, from (6) (6) (17) and (8) we see that $\omega_{2 r+1,2 r+3}(E), r \leq n-1$, cannot vanish. This completes the inductive argument and proves (14) and (15). 
¿From Fact 9 the polar surface $g_{\theta}^{*}$ to $g_{\theta}$ is isometric to $g^{*}$ for any $\theta \in \mathcal{M}(g)$. It follows from (15) that the polar surfaces $g_{\theta_{j}}^{*}=\phi_{\theta_{j}} e_{2 n+1}: L^{2} \backslash L_{0} \rightarrow \mathbb{S}^{2 n+1}$ satisfy

$$
\sum_{j=1}^{m} e^{i \theta_{j}}\left\langle g_{\theta_{j}}^{*}, v_{j}\right\rangle=0 .
$$

We easily see that

$$
\sum_{j=1}^{m-1}\left\langle g_{\theta_{j}}^{*}, v_{j}^{1}\right\rangle=0
$$

where $v_{j}^{1}=\sin \left(\theta_{m}-\theta_{j}\right) v_{j} \neq 0$. We have from Fact 9 that the surfaces $g_{\theta_{j}}^{*}, 1 \leq j \leq m-1$, are isotropic. If $m>2$ and since the polar surface of $g_{\theta_{j}}^{*}$ is just $g_{\theta_{j}}$, arguing as for (16) we obtain that

$$
\sum_{j=1}^{m-2}\left\langle g_{\theta_{j}}, v_{j}^{2}\right\rangle=0
$$

where $v_{j}^{2}=\sin \left(\theta_{m-1}-\theta_{j}\right) v_{j}^{1} \neq 0$. By repeating the argument, if necessary, we conclude that either $\left\langle g_{\theta_{1}}, v\right\rangle=0$ or $\left\langle g_{\theta_{1}}^{*}, v\right\rangle=0$ for some $v \neq 0$. But from the definition of the associated family we have that any $g_{\theta}$ is substantial. That any $g_{\theta}^{*}$ must also be substantial follows easily using Proposition 8 in [9]. Thus, we reached a contradiction and the claim has been proved.

We now conclude the proof. According to Theorem 1 the set $\mathcal{M}(g)$ is either finite or $\mathbb{S}^{1}$. Suppose that $\mathcal{M}(g)=\mathbb{S}^{1}$. Then any finite subset of height functions of the surfaces $g_{\theta}, \theta \in \mathbb{S}^{1}$, must be linearly independent. But since these functions are eigenfunctions of the Laplace operator on $L^{2}$ with eigenvalue 2, this contradicts the fact that any eigenspace of the Laplace operator on a compact manifold has finite dimension.

Proof of Corollary 3: From Theorem 2 we know that $\mathcal{M}(g)$ is finite. Consider the isotropic immersions $g^{t}=g \circ \varphi_{t}$. Since the second fundamental form of $g^{t}$ depends continuously on the parameter, we deduce that $g^{t}$ for any $t$ is congruent to exactly one $g_{\theta}$ for some $\theta \in \mathcal{M}(g)$. Since $\varphi_{0}=i d$, by continuity we conclude that $g^{t}$ is congruent to $g$ for any $t$.

\section{References}

[1] J. Barbosa, On minimal immersions of $\mathbb{S}^{2}$ into $\mathbb{S}^{2 m}$, Trans. Amer. Math. Soc. 210 (1975), 75-106.

[2] J. Bolton, L. Vranken and L. Woodward, On almost complex curves in the nearly Kähler 6-sphere, Quart. J. Math. Oxford 45 (1994), 407-427. 
[3] J. Bolton and L. Woodward, Congruence theorems for harmonic maps from a Riemann surface into $\mathbb{C P}^{n}$ and $\mathbb{S}^{n}$. J. London Math. Soc. 45 (1992), 363-376.

[4] J. Bolton, F. Pedit and L. Woodward, Minimal surfaces and the affine Toda field model, J. reine angew. Math. 459 (1995), 119-150.

[5] R. Bryant, Submanifolds and special structures on the octonions, J. Differential Geom. 17 (1982), 185-232.

[6] E. Calabi, Minimal immersions of surfaces in Euclidean spheres, J. Differentil Geom. 1 (1967), 111-125.

[7] C.C. Chen, The generalized curvature ellipses and minimal surfaces. Bull. Acad. Sinica 11 (1983), 329-336.

[8] S.S. Chern, On the minimal immersions of the two-sphere in a space of constant curvature, Problems in Analysis, 27-40. University Press, Princeton (1970).

[9] M. Dajczer and L. Florit, A Class of austere submanifolds, Illinois Math. J. 45 (2001), 735-755.

[10] M. Dajczer and D. Gromoll, Real Kaehler submanifolds and uniqueness of the Gauss map, J. Differential Geom. 22 (1985), 13-28.

[11] M. Dajczer and Th. Vlachos, The associated family of an elliptic surface and applications to minimal submanifolds. To appear in Geometriae Dedicata.

[12] J.H. Eschenburg and Th. Vlachos, Pseudoholomorphic Curves in $\mathbb{S}^{6}$ and the Octonions, Preprint.

[13] H. Hashimoto, T. Taniguchi and S. Udagawa, Constructions of almost complex 2-tori of type (III) in the nearly Kaehler 6-sphere, Differential Geom. Appl. 21 (2004), 127-145.

[14] G. Johnson, An intrinsic characterization of a class of minimal surfaces in constant curvature manifolds, Pacific J. Math. 149 (1991), 113-125.

[15] R. Miyaoka, The family of isometric superconformal harmonic maps and the affine Toda equations, J. reine angew. Math. 481 (1996), 1-25.

[16] J. Ramanathan, Rigidity of minimal surfaces in $\mathbb{S}^{3}$, Manusucripta Math. 60 (1988), 417-422.

[17] Th. Vlachos, Congruence of minimal surfaces and higher fundamental forms, Manuscripta Math. 110 (2003), 77-91. 
[18] Th. Vlachos, Minimal surfaces, Hopf differentials and the Ricci condition. Manuscripta Math. 126 (2008), 201-230.

[19] Th. Vlachos, Exceptional minimal surfaces in spheres. See arXiv:1108.5834.

IMPA - Estrada Dona Castorina, 110 22460-320 - Rio de Janeiro - Brazil

E-mail: marcos@impa.br
Univ. of Ioannina - Math. Dept. 45110 Ioannina - Greece

E-mail: tvlachos@uoi.gr 